\title{
Glibenclamide transfer across the perfused human placenta is determined by albumin binding not transporter activity
}

\author{
Emma M. Lofthouse ${ }^{1,3}$, Jane K. Cleal ${ }^{1,3}$, Georgina Hudson ${ }^{1}$, Rohan M. Lewis ${ }^{1,3}$, \\ Bram G. Sengers ${ }^{2,3}$
}

\author{
Affiliations: \\ 1. University of Southampton, Faculty of Medicine \\ 2. University of Southampton, Faculty of Engineering and Physical Sciences \\ 3. Institute for Life Sciences, University of Southampton, UK
}

\begin{abstract}
The placenta mediates the transfer of maternal nutrients into the fetal circulation while removing fetal waste products, drugs and environmental toxins that may otherwise be detrimental to fetal development. This study investigated the role of drug transporters and protein binding in the transfer of the antidiabetic drug glibenclamide across the human placental syncytiotrophoblast using placental perfusion experiments and computational modelling. In the absence of albumin, placental glibenclamide uptake from the fetal circulation was not affected by competitive inhibition with bromosulphothalein (BSP), indicating that OATP2B1 does not mediate placental glibenclamide uptake from the fetus. In the presence of maternal and fetal albumin, BSP increased placental glibenclamide uptake from the fetal circulation by displacing glibenclamide from BSA, increasing the free fraction of glibenclamide driving diffusive transport. The P-gp and BCRP inhibitor GF120918 did not affect placental glibenclamide uptake from the maternal circulation and as such this study did not find any evidence for the apical efflux transporters in placental glibenclamide transfer. Computational modelling confirmed that albumin binding and not transporter activity, is the dominant factor in the transfer of glibenclamide across the human placenta. The effect of BSP binding to albumin on promoting the diffusive transfer of glibenclamide highlights the importance of drug-protein binding interactions and their interpretation using computational modelling.
\end{abstract}

\section{Keywords:}

human placenta, glibenclamide, albumin, OATP and ABC transporters 


\section{INTRODUCTION}

The placenta as the interface between the mother and developing fetus mediates the transfer of maternal nutrients into the fetal circulation while simultaneously removing fetal waste products and protects the fetus from xenobiotics [1]. Many women require medication during pregnancy, so from a safety perspective it is important to understand the pharmacokinetics of placental drug transport in order to understand the factors affecting fetal exposure.

The human placenta has been reported to express a variety of transport proteins, including ATP binding cassette $(\mathrm{ABC})$ transporters such as P-glycoprotein (P-gp, MDR1, $A B C B 1$ ), breast cancer resistance protein (BCRP, $A B C G 2)$ and the multidrug resistance-associated proteins 1-3 (MRP, ABCC1-3) [2, 3] that could help clear drugs and toxins back to the maternal circulation (Fig. 1A). Both P-gp and BCRP are highly expressed in the placenta $[4,5]$. There is conflicting evidence regarding MRP isoform expression on the placental syncytiotrophoblast. MRP2 expression was demonstrated on the apical microvillous membrane (MVM) alone via immunohistochemistry and western blotting [6]. MRP1 and MRP3 were also found to be expressed primarily on the fetal endothelium with lower expression on the MVM [6]. However, other studies have been unable to detect MRP3 expression in the placenta [7] and it has been reported that MRP1 is only expressed on the basal membrane (BM) of the syncytiotrophoblast [8].

To pass between the maternal and fetal circulation any drug would need to cross both the MVM and BM of the placental syncytiotrophoblast. Transporters such as the organic anion-transporting polypetide OATP2B1 (SLCO2B1) on the BM are thought to work together with $\mathrm{ABC}$ efflux transporters on the MVM to effect clearance of certain drugs from the fetal circulation back to the maternal circulation $[2,9]$. In addition, OATP4A1 (SLCO4A1) is expressed on the MVM [10] and could play a role in bidirectional transfer.

Gestational diabetes is associated with increased fetal macrosomia, neonatal hypoglycemia and perinatal complications [11, 12]. Maternal treatment for this condition consists of dietary modifications and if this proves insufficient, medication with insulin or drugs is required [11, 13]. Glibenclamide is a sulfonylurea drug that has 
been used to treat gestational diabetes. Initial concerns about glibenclamide transfer to the fetus were quelled by reports that measured concentrations in the fetal circulation were very low, even at maternal concentrations significantly higher than therapeutic $[11,14,15]$. However, glibenclamide has been found less effective than metformin based on a range of pregnancy outcomes [13]. A high variability in umbilical vein glibenclamide concentrations has been observed in vivo, which was attributed to intersubject variability in the function of placental transporters, although it is unclear to what extent this could be explained by the wide range in dose and sampling time [11].

Low transfer of glibenclamide across the human placenta has been attributed to high plasma protein binding, in combination with a short half-life, while binding to the placenta itself could also play a role [12]. It is thought that only the free fraction of glibenclamide can be transported across a cell membrane and it has been reported that under physiological conditions, the free fraction of glibenclamide is only $0.2 \%[12,16]$. Ex vivo human placental perfusion studies have demonstrated significantly higher glibenclamide transfer in the fetal to maternal direction compared to the maternal to fetal direction, consistent with the activity of efflux pumps on the MVM [17]. This was attributed to BCRP, as opposed to P-gp or MRPs, based on studies using the BCRP inhibitor nicardipine and the lack of effect of the P-gp inhibitor verapamil and the MRP inhibitor indomethacin, combined with the low expression of MRPs [7, 17, 18]. Alternatively, such asymmetry in transfer could result from differences in maternal and fetal protein binding $[16,19]$.

In vitro studies using human placental MVM vesicles and non-placental cell lines have concluded that glibenclamide is transported by BCRP, with conflicting data on the role of MRPs in vesicles [3, 20,21]. Depending on concentration, glibenclamide transfer was determined by the interplay between BCRP and passive diffusion in rat placental perfusions [22]. Both BCRP and OATP2B1 are highly expressed in the placenta [9], with reported $\mathrm{Km}$ values of $13.7 \mu \mathrm{M}$ [23] and $6.26 \mu \mathrm{M}$ [24] for glibenclamide, respectively. Previous studies have demonstrated the interactions between BCRP on the MVM and OATP2B1 on the BM in promoting basal to apical transfer of steroid sulphates in vitro $[9,25]$. Although glibenclamide is an OATP2B1 substrate, only limited glibenclamide uptake ratios of 1.5-2.7 compared to control have been found for HEK293 cells transfected to express OATP2B1 [26-28], which appears to be due to the 
high diffusive uptake in controls. The role of OATPs on the BM in the fetal to maternal transfer of glibenclamide has not been investigated in placental perfusion studies.

This study aimed to determine the role of drug transporters and protein binding in the transfer of glibenclamide across the human placental syncytiotrophoblast using placental perfusion experiments and computational modelling.

\section{METHODS}

Term human placentas were collected with written informed consent from women delivering at the Princess Anne Hospital in Southampton with approval from the Southampton and Southwest Hampshire Local Ethics Committee (11/SC/0529). All placentas used in this study were obtained from uncomplicated pregnancies delivered at term by caesarean section.

Placentas were perfused using the methodology of Schneider et al. [29] as adapted in our laboratory [30]. Both the maternal and fetal circulation were operated in open-loop configuration (non-recirculating). Placentas were perfused with Earle's Bicarbonate Buffer (EBB: $5 \mathrm{mM}$ glucose, $1.8 \mathrm{mM} \mathrm{CaCl}_{2}, 0.4 \mathrm{mM} \mathrm{MgSO}_{4}, 116.4 \mathrm{mM} \mathrm{NaCl}, 5.4$ $\mathrm{mM} \mathrm{KCl}, 26.2 \mathrm{mM} \mathrm{NaHCO} 3,0.9 \mathrm{mM} \mathrm{NaH}_{2} \mathrm{PO}_{4}$ ), gassed with $5 \% \mathrm{CO}_{2}$ and $95 \% \mathrm{O}_{2}$. Perfusion via the fetal catheter into a chorionic plate fetal artery was performed at 6 $\mathrm{ml} / \mathrm{min}$ and via 5 maternal catheters at $14 \mathrm{ml} / \mathrm{min}$ using a roller pump. Before starting the experiments detailed below, a washout step was included whereby the fetal and maternal circulation of every placenta was perfused with EBB for 15 minutes. Throughout the experiments, fetal and maternal placental circulation pressures were recorded using Chart v4.2., Approximately $1.5 \mathrm{ml}$ of venous exudate was collected from maternal and fetal venous outflows at regular intervals.

${ }^{3}$ H-glibenclamide (Packard-Perkin Elmer, Massachusetts USA, see Supplementary data) and creatinine (a marker of paracellular diffusion) were added to the fetal or maternal circulations and levels in the fetal and maternal effluent analysed via liquid scintillation counting, using 5 min counts to maximise counting efficiency (Packard-Perkin Elmer, Massachusetts USA) and the creatinine infinity reagent kit (ThermoFisher), respectively. At the end of the experiment, the perfused region of placental cotyledon 
(the white region cleared of blood) was obtained by trimming off the non-perfused tissue, then blotted and weighed.

\section{Fetal to maternal ${ }^{3} \mathrm{H}$-glibenclamide, BSP and MK571 perfusions}

The fetal circulation of isolated cotyledons was perfused with EBB containing $1 \mathrm{~g} / \mathrm{L}$ bovine serum albumin (BSA), $1.8 \mathrm{mmol} / \mathrm{L}$ creatinine and ${ }^{3} \mathrm{H}$-glibenclamide (93 $\mathrm{pmol} / \mathrm{L}$ ) for $30 \mathrm{~min}$ before $150 \mu \mathrm{mol} / \mathrm{L}$ bromosulphothalein (BSP, an OATP substrate and inhibitor) was added for a further $30 \mathrm{~min}$. The fetal buffer was then returned to EBB containing $1 \mathrm{~g} / \mathrm{L}$ BSA, $1.8 \mathrm{mM}$ creatinine and ${ }^{3} \mathrm{H}$-glibenclamide for $30 \mathrm{~min}$ before $43 \mu \mathrm{M}$ MK571 (an MRP inhibitor) was added for a final 30 min block. Throughout these changes, the maternal circulation was perfused with EBB containing $1 \mathrm{~g} / \mathrm{L} \mathrm{BSA}$ alone. The protocol was then repeated in the absence of BSA in either circulation

\section{Alternating fetal to maternal / maternal to fetal ${ }^{3} \mathrm{H}$-glibenclamide perfusions}

The fetal and maternal circulations of isolated cotyledons were perfused with alternating EBB containing $1 \mathrm{~g} / \mathrm{L}$ BSA alone and EBB containing ${ }^{3} \mathrm{H}$-glibenclamide (93 pmol/L), $1 \mathrm{~g} / \mathrm{L}$ BSA and $1.8 \mathrm{mmol} / \mathrm{L}$ creatinine for 30 minutes before repeating these conditions using $2 \mu \mathrm{mol} / \mathrm{L}$ GF120918 (a P-gp and BCRP inhibitor), added to the buffer with ${ }^{3} \mathrm{H}$-glibenclamide only. The protocol was then repeated in the absence of BSA in either circulation.

\section{Maternal to fetal BSA free ${ }^{3} \mathrm{H}$-glibenclamide perfusions}

The maternal circulation of the cotyledon was perfused with EBB containing ${ }^{3} \mathrm{H}$ glibenclamide (46 pmol/L) and $1.8 \mathrm{mmol} / \mathrm{L}$ creatinine for $60 \mathrm{~min}$ before $2 \mu \mathrm{mol} / \mathrm{L}$ GF120918 was added for a further $60 \mathrm{~min}$. The maternal buffer was then returned to EBB containing ${ }^{3} \mathrm{H}$-glibenclamide and $1.8 \mathrm{mM}$ creatinine for a further 60 minutes. Throughout the experiment, the fetal circulation was perfused with BSA free EBB.

\section{${ }^{3} \mathrm{H}$-glibenclamide protein binding}

To determine the free fraction of glibenclamide with and without the presence of BSA, ${ }^{3} \mathrm{H}$-glibenclamide $(1.8 \mathrm{nmol} / \mathrm{L})$ in EBB alone, EBB containing $1 \mathrm{~g} / \mathrm{l} \mathrm{BSA}$ or EBB containing $1 \mathrm{~g} / \mathrm{l} \mathrm{BSA}+100 \mu \mathrm{mol} / \mathrm{L}$ BSP (5-6 replicates per condition, except 3 for EBB + BSP alone) was dialysed against $100 \mu$ EBB (BSA free) using a Slide-a-lyzer 
$7 \mathrm{~K}$ dialysis membrane for $24 \mathrm{~h}$ at room temperature on a rocker (Fisher Scientific). Transfer of ${ }^{3} \mathrm{H}$-glibenclamide into the column was analysed by liquid scintillation counting (Packard-Perkin Elmer, Massachusetts USA). Trial experiments at 8,24 and $48 \mathrm{hr}$ showed that equilibrium was reached by 24 hours.

To determine the free fraction of ${ }^{3} \mathrm{H}$-glibenclamide in perfusate experiments, 1.3 $\mathrm{ml}$ samples from fetal and maternal perfusate (3-4 replicates, consisting of two pooled samples, per condition for each placenta) were dialysed against $100 \mu \mathrm{L}$ EBB (BSA free) using the same protocol. Protein in perfusate samples collected (n =6-8 per condition for each placenta) was analysed using the Pierce BCA protein assay kit (ThermoFisher).

\section{Statistics}

Protein concentrations and ${ }^{3} \mathrm{H}$-glibenclamide free fractions from the dialysis with and without BSA were analysed by two-way ANOVA followed by Tukey-Kramer post hoc test where necessary to distinguish effects and interactions. Significance was assumed at $\mathrm{p} \leq 0.05$ and all data are presented as the mean \pm standard deviation of three placentas, calculated using the average value of the samples for each placenta as outlined in the previous section.

\section{Computational model}

Glibenclamide transfer was described using a compartmental model for placental transfer described previously [31,32]. Separate maternal, syncytiotrophoblast and fetal compartments were distinguished (Fig. 1B). Fluxes between compartments were assumed to be governed by simple diffusion of the free, unbound, glibenclamide fraction as described by Eqs. 1-3. No effects of transporters were incorporated in first instance.

$$
\begin{array}{ll}
\frac{\mathrm{d} c_{m}}{\mathrm{~d} t}=\frac{1}{V_{m}}\left(P S_{M V M}\left(f_{u s} c_{s}-f_{u m} c_{m}\right)+Q_{m}\left(c_{i n m}-c_{m}\right)\right) & \text { Eq. } 1 \\
\frac{\mathrm{d} c_{s}}{\mathrm{~d} t}=\frac{1}{V_{s}}\left(P S_{M V M}\left(f_{u m} c_{m}-f_{u s} c_{s}\right)+P S_{B M}\left(f_{u f} c_{f}-f_{u s} c_{s}\right)\right) & \text { Eq. } 2 \\
\frac{\mathrm{d} c_{f}}{\mathrm{~d} t}=\frac{1}{V_{f}}\left(P S_{B M}\left(f_{u s} c_{s}-f_{u f} c_{f}\right)+Q_{f}\left(c_{i n f}-c_{f}\right)\right) & \text { Eq. } 3
\end{array}
$$


Here $c[\mathrm{~mol} / \mathrm{L}]$ are the concentrations in the maternal, syncytiotrophoblast and fetal compartments, indicated by subscripts $\mathrm{m}, \mathrm{s}$, and $\mathrm{f}$, respectively, while $V[\mathrm{~L}]$ are the compartment volumes. $c_{\text {inm }}$ and $c_{\text {inf }}$ are the arterial inlet concentrations in the maternal and fetal compartments. $P S_{M V M}$ and $P S_{B M}[\mathrm{~L} / \mathrm{min}]$ are the overall cotyledon microvillous membrane (MVM) and basal membrane (BM) permeability surface area products. The free fractions in each compartment are given by $f_{u}[-]$, assumed constant here. $Q[\mathrm{~L} / \mathrm{min}]$ are the perfusion flow rates for the maternal and fetal circulation.

Creatinine transfer was modelled based on paracellular diffusion directly from the maternal to the fetal compartment, bypassing the syncytiotrophoblast compartment, with free fractions equal to 1 (Supplementary data A).

The model was implemented in Matlab (R2018a, The Mathworks, Natick, MA, USA). Eqs. 1-3 where solved numerically using the 'ode45' function (Runge-Kutta $(4,5)$ method). For simplicity, the measured cotyledon weight in $[\mathrm{kg}]$ was equated to the total volume in [L]. The volume $V$ of the individual compartments was then calculated based on relative volume fractions of $34 \%, 15 \%$ and $7.4 \%$ for the maternal, syncytiotrophoblast and fetal compartments, respectively, as in our previous work [32, 33]. Measured fetal flow rates were used to account for any variation during the different phases of the experiment.

The model was fitted to the experimental data based on the sum of the normalised least square errors for the maternal and fetal concentrations, using the error criterion $E_{\text {tot }}$ defined in Eq. 4.

$$
E_{t o t}=\frac{\sum\left(c_{m}^{i}-c_{m \text { exp }}^{i}\right)^{2}}{\sum\left(c_{m \text { exp }}^{i}\right)^{2}}+\frac{\sum\left(c_{f}^{i}-c_{f \text { exp }}^{i}\right)^{2}}{\sum\left(c_{f \text { exp }}^{i}\right)^{2}}
$$

$c_{m \text { exp }}^{i}$ are the experimental concentrations measured and $c_{m}^{i}$ the computational values at time points $i$, consisting of the last 4 time points of every experimental phase in order to focus on steady state behaviour. Parameter estimation was implemented using the Matlab 'fminsearch' function, running the complete model within the parameter estimation loop. Unless stated otherwise, in general three parameters were fitted: The MVM and BM permeability surface area products $P S_{M V M}, P S_{B M}$ and the 
syncytiotrophoblast free fraction $f_{u s}$. The effective permeability surface area product of the two membranes in series was calculated based on the estimated parameters as $P S_{e f f}=\left(P S_{M V M} P S_{B M}\right) /\left(P S_{M V M}+P S_{B M}\right)$.

Initial parameter estimates were varied to verify the uniqueness of the solution and a sensitivity analysis was conducted in which the volume of individual compartments was either reduced by a factor 10 or increased to the maximum remaining volume.

\section{Glibenclamide-BSA binding}

The theoretical glibenclamide free fraction in presence of BSA was calculated analytically (Supplementary data B). The dissociation constant used for glibenclamide binding to BSA was $K_{d g l b}=6.7 \times 10^{-7} \mathrm{~mol} / \mathrm{L}$, and a single binding site was assumed for consistency [34]. The BSA molecular weight was $66.5 \mathrm{kDa}$.

The theoretical glibenclamide free fraction in presence of both BSA and BSP was determined numerically by solving the multiple substrate binding problem (Supplementary data C), assuming a single BSA binding site and that both glibenclamide and BSP can bind to BSA, but not simultaneously. The value of the dissociation constant for BSP used was $K_{d B S P}=3.8 \times 10^{-5} \mathrm{~mol} / \mathrm{L}$, based on the association constant for human serum albumin [35].

\section{RESULTS}

Note that in the following, the terms 'uptake' and 'transfer' were used to describe the disappearance and appearance of a solute in the respective circulations, without necessarily implying the involvement of active and/or transporter mediated processes.

\section{Fetal side ${ }^{3} \mathbf{H}$-glibenclamide perfusion}

Results for the fetal to maternal glibenclamide perfusion experiment are given in Fig. 2 ( $\mathrm{n}=3$ cotyledons, mean weight $63 \pm 27 \mathrm{~g}$ ). Removal of BSA from the perfusion buffer increased the uptake of ${ }^{3} \mathrm{H}$-glibenclamide from the fetal circulation (Fig. 2A). 
Transfer of glibenclamide to the maternal circulation was also increased in the absence of BSA, although the time scale of the response was slower (Fig. 2B).

In the presence of BSA, addition of BSP led to an increase in fetal uptake of glibenclamide and its transfer to the maternal circulation, but BSP did not have an effect on glibenclamide transfer in the absence of BSA. Addition of the MRP inhibitor MK571 did not affect glibenclamide uptake from the fetal circulation nor transfer to the maternal circulation.

Theoretical predictions for the glibenclamide free fraction in the perfusate as a function of BSA concentration, are shown in Fig. 3A. It can be seen that the free fraction becomes independent of glibenclamide concentration at very low concentrations of glibenclamide, such as those for the tracer used in the present study $(93 \mathrm{pmol} / \mathrm{L})$. A free fraction of $4.3 \%$ was predicted to correspond to the $1 \mathrm{~g} / \mathrm{L} \mathrm{BSA}$ used in the experiment. Addition of $150 \mu \mathrm{mol} / \mathrm{L} \mathrm{BSP}$, in combination with $1 \mathrm{~g} / \mathrm{L} \mathrm{BSA}$, was predicted to increase the glibenclamide free fraction to $17 \%$ (Fig. 3B).

The computational model was fitted to the average fetal and maternal vein glibenclamide concentrations (Fig. 2), under the assumption that the glibenclamide free fraction was $100 \%$ in buffer without BSA, while estimating the free fractions in buffer with BSA. This resulted in an estimated free fraction of $31 \%$ for buffer with BSA, and a free fraction of $93 \%$ for buffer containing both BSA and BSP.

The ${ }^{3} \mathrm{H}$-glibenclamide free fraction in EBB, measured using dialysis, was $21 \pm 3 \%$ for buffer with BSA and $54 \pm 12 \%$ for buffer containing both BSA and BSP (Fig. 4). Statistical analysis confirmed an interaction between BSP and BSA (two-way ANOVA, $\mathrm{p}=0.0018, \mathrm{n}=3-6)$. There was no difference in the glibenclamide free fraction for buffer with BSP $(92 \pm 14 \%)$ or without BSP $(96 \pm 12 \%)$ in the absence of BSA ( $p=$ 0.93, Tukey-Kramer). Similar results were found for the actual fetal perfusate from the experiment (Fig. 4). The measured free fraction of glibenclamide was $23 \pm 7 \%$ for perfusate with BSA alone, and $50 \pm 10 \%$ for perfusate containing BSA and BSP. There was an interaction between BSP and BSA (two-way ANOVA, $p=0.014, n=3$ ). No difference in the glibenclamide free fraction was found for perfusate with BSP (82 \pm $12 \%)$ or without BSP $(89 \pm 8 \%)$ in absence of BSA $(p=0.82$, Tukey-Kramer). 
The model fit of the average glibenclamide concentrations was repeated based on the free fractions in fetal perfusate measured using dialysis. The maternal glibenclamide free fractions were assumed to be the same as the fetal perfusate values in absence of BSP. This produced results similar to the experimental data (Fig. 2), but underestimated uptake from the fetal circulation for BSP in combination with BSA. The estimated effective permeability surface area product was $P S_{\text {eff }}=4.2 \times 10^{-3} \mathrm{~L} / \mathrm{min}$ and tissue free fraction $f_{u t i s s}=1.3 \%$. The estimated permeability surface area product for creatinine was $P S_{\text {crea }}=1.5 \times 10^{-3} \mathrm{~L} / \mathrm{min}$.

\section{Alternating fetal / maternal ${ }^{3} \mathrm{H}$-glibenclamide perfusion}

Results for the alternating fetal-maternal glibenclamide perfusion experiments are shown in Fig. 5 ( $\mathrm{n}=3$ cotyledons, weight $30 \pm 10 \mathrm{~g}$ ). It can be observed that there is increased placental glibenclamide uptake from either the fetal or maternal side in the absence of BSA, as evident from the lower vein concentrations. No effects of the P-gp and BCRP inhibitor GF120918 were apparent.

The free fractions of ${ }^{3} \mathrm{H}$-glibenclamide for the alternating fetal-maternal experiment are shown in Fig. 6A. Measured glibenclamide free fractions in fetal and maternal perfusate without BSA were $82 \pm 3 \%$ and $73 \pm 3 \%$, respectively. Two-way ANOVA with post hoc testing using Tukey-Kramer did not find a difference between fetal and maternal free fractions in absence of BSA $(p=0.12)$. Measured protein concentrations in the corresponding samples are reported in Fig. 6B. There was no difference between fetal and maternal protein in absence of BSA ( $p=0.99$, two-way ANOVA with TukeyKramer post hoc).

The model was fitted to the perfusion data using the average glibenclamide free fractions from the dialysis for each circulation, with or without BSA. Model results for fitting the average glibenclamide concentrations are shown in Fig. 5. The estimated parameters based on fitting the results for individual cotyledons were as follows: Maternal side permeability surface area product $P S_{M V M}=1.6 \pm 0.6 \times 10^{-2} \mathrm{~L} / \mathrm{min}$, fetal side $P S_{B M}=5 \pm 4 \times 10^{-3} \mathrm{~L} / \mathrm{min}$, and free fraction in the tissue $f_{u \text { tiss }}=0.8 \pm 0.3 \%$. The overall effective permeability surface area product for glibenclamide was calculated as 
$P S_{\text {eff }}=3.5 \pm 1.3 \times 10^{-3} \mathrm{~L} / \mathrm{min}$. The estimated permeability surface area product for creatinine based on average concentrations was $P S_{\text {crea }}=3.0 \times 10^{-3} \mathrm{~L} / \mathrm{min}$.

\section{Maternal side ${ }^{3} \mathbf{H}$-glibenclamide perfusion}

The results of the maternal to fetal glibenclamide perfusion experiments without BSA are shown in Fig. $7(\mathrm{n}=3$ cotyledons, weight $45 \pm 6$ g). Fetal vein concentrations revealed a slow increase in transfer of glibenclamide to the fetal circulation over time before reaching steady state after $2.5 \mathrm{~h}$. The tissue accumulation rate of glibenclamide gradually reduced over a similar timescale, from approximately $50 \%$ of maternal glibenclamide input at the start of the experiment to zero towards the end, based on the mass balance calculated from the maternal and fetal placental arterial-venous differences and flow rates. No effects of the inhibitor GF120918 were apparent.

The measured glibenclamide free fraction in maternal perfusate containing no BSA was $85 \pm 4 \%$ and corresponding protein concentration $0.22 \pm 0.08 \mathrm{~g} / \mathrm{L}$ at steady state. For modelling the results it was assumed that the fetal glibenclamide free fraction was the same as the measured maternal value. The model fit based on the average concentrations is shown in Fig. 7, while the fits of individual cotyledons yielded an overall effective permeability surface area product for glibenclamide $P S_{\text {eff }}=2.7 \pm 1.5$ $\times 10^{-3} \mathrm{~L} / \mathrm{min}$ and free fraction in the tissue $f_{u \text { tiss }}=1.2 \pm 0.8 \%$. The estimated permeability surface area product for creatinine was $P S_{\text {crea }}=2.3 \pm 0.8 \times 10^{-3} \mathrm{~L} / \mathrm{min}$.

\section{Discussion}

This study demonstrates the importance of plasma protein binding in determining placental transfer of the anti-diabetic drug glibenclamide. No transporter mediated transfer could be demonstrated suggesting that transporters play little or no role in glibenclamide transfer in the human placenta.

\section{Transporters}

First, the role of basal membrane transporters in mediating placental glibenclamide uptake from the fetal circulation was investigated. In the absence of BSA, placental glibenclamide uptake from the fetal circulation was not affected by competitive 
inhibition with the OATP substrate BSP, indicating that OATP2B1 does not mediate placental glibenclamide uptake from the fetus.

In the presence of BSA, BSP was shown to increase placental glibenclamide uptake from the fetal circulation (Fig. 2), however no effect of BSP was observed when BSA was removed from the buffer. OATP2B1 is highly expressed in the basal membrane [9], so these negative results would suggest a relatively low uptake rate/affinity for glibenclamide compared to passive diffusion, in accordance with the modest uptake compared to control observed in HEK293 cells transfected to express OATP2B1 [2628]. In line with previous studies [7], no effect of the MRP inhibitor MK571 on placental glibenclamide uptake from the fetal circulation was observed, further excluding the possibility that the increased placental glibenclamide uptake in presence of BSP was related to inhibition of efflux transporters on the basal membrane.

Secondly, the contribution of $\mathrm{ABC}$ transporters on the apical microvillous membrane was investigated. The P-gp and BCRP inhibitor GF120918 has been used successfully in rat placenta perfusion experiments to block transport for low glibenclamide concentrations, while diffusion became dominant at higher concentrations [22]. However, despite the low glibenclamide tracer concentrations and using the same inhibitor concentration, no effects of GF120918 were observed for human placenta in the current study with short (Fig. 5) or longer term exposure (Fig. 7). As such this study did not find any evidence for the apical efflux transporters in placental glibenclamide transfer. A limitation of the current study is that we could not measure the actual concentrations of inhibitors entering the placenta in light of potential binding to the tubing of the perfusion setup, which can be an important factor in perfusion experiments [36], although this was mitigated by the open loop configuration employed under steady state conditions. While this was not confirmed directly, no significant effects of binding to the tubing were apparent in the results for glibenclamide, considering that the vein concentrations were close to stock in specific cases, as well as the correspondence between uptake and transfer across the placenta.

Computational modelling of the alternating fetal-maternal perfusion data confirmed that the results could be represented based on simple diffusion, with the same permeability in both directions and no additional transporter contributions (Fig. 5). This finding is inconsistent with work in rodents [22], suggesting that there may be species 
differences. There is considerable overlap between inhibitors of individual $\mathrm{ABC}$ and OATP transports, depending on concentration [37, 38]. This adds to the complexities of correctly interpreting and comparing the results of inhibitor studies in different species, which do not necessarily express the same transporters and highlights the importance of performing safety studies in human placenta.

\section{Protein binding}

To explain the stimulatory effect of BSP on placental glibenclamide transfer it was hypothesised that the BSP was displacing glibenclamide from BSA, increasing the free fraction of glibenclamide driving diffusive transport. Our initial theoretical predictions and model estimates based on the experimental data supported this hypothesis, but indicated that the free fractions needed to explain the data were higher than expected from binding affinities in the literature [34].

Experimental measurements using dialysis were consistent with model estimates. Multiple binding sites have been distinguished for glibenclamide and normal human serum albumin, with dissociation constants of $7 \times 10^{-7}$ and $2 \times 10^{-5} \mathrm{~mol} / \mathrm{L}$ [39]. The

high affinity value is equivalent to the $K_{d} g l b=6.7 \times 10^{-7} \mathrm{~mol} / \mathrm{L}$ for BSA [34] that was used for the theoretical predictions, while the $K_{d ~ g l b}=3.9 \times 10^{-6} \mathrm{~mol} / \mathrm{L}$ calculated for $\mathrm{EBB}+\mathrm{BSA}$ from the current data (Supplementary data C) lies somewhere in between, rather than approaching the high affinity value as may have been expected based on the low tracer concentrations used. This cannot be explained from the fact that the current binding studies were carried out at room temperature as the glibenclamide binding affinity to albumin decreases with increasing temperature [40].

Measured protein concentrations displayed a rapid drop after the switch over to buffer without BSA and no time delays due to the effects of mixing were apparent within the sampling window.

Consistent with previous studies [16], an initial slow release of native protein from the placenta was observed in the maternal circulation for the BSA free experiments, which could take up to $2 \mathrm{~h}$ to reach steady state (data not shown). Nanovskaya et al. observed differences between maternal and fetal glibenclamide binding associated with the release of native protein during closed loop perfusion experiments [16, 19]. Our 
simulations of their experiments confirm how such differences in binding could give rise to an apparent asymmetry in transfer, which is lost as more albumin is added equally to both circulations (Supplementary data E). It remains to be seen to what extent the modest reduction in asymmetry following addition of the BCRP inhibitor Nicardipine to the maternal circulation [18] could be explained alternatively by binding interactions with native protein, similar as observed for BSP and BSA here.

In the current study, native protein levels remained low due to the absence of recirculation in open loop configuration and no differences between the glibenclamide free fraction in maternal and fetal perfusate were found consistently across all experiments in absence of BSA. While native placental protein release may affect drug transfer in perfusion systems it is unlikely to be physiologically important in vivo where the levels of albumin and other proteins will be much higher. In this respect, it has to be noted that no difference in the percentage unbound was found for glibenclamide between maternal and umbilical cord vein plasma in vivo [41].

\section{Tissue accumulation}

Glibenclamide could accumulate in tissue bound to intracellular proteins. Following switches between experimental conditions, the maternal and fetal vein glibenclamide concentrations appeared to reach quasi steady state within the experimental time blocks, but nonetheless a slow increase in venous concentrations over longer time scales was apparent (Fig. 2, 5 and 7), which needs to be considered in any comparisons of different experimental designs. As predicted by the model, these increases in output concentrations observed were due to the gradual tissue accumulation of ${ }^{3} \mathrm{H}$ glibenclamide over time, leading to increased tissue concentrations mediating transfer. In the maternal to fetal perfusion experiment without BSA (Fig. 7), the experimental mass balance showed an initial high rate of tissue accumulation (50\%) and took over two hours to approach steady state, indicating the high binding capacity of the tissue. Note that, despite the similar timescale, this cannot be explained based on the initial presence of any native plasma protein in the maternal perfusate as uptake decreases rather than increases.

The model estimated a glibenclamide tissue free fraction of approximately $1 \%$, which is in the same order of magnitude as found previously, e.g. 3\% for liver cells [42]. 
Importantly, this estimated tissue free fraction is directly related to the relevant volume fraction assumed in the model, in this case for the syncytiotrophoblast. If tissue binding occurs in a larger tissue volume then the estimated free fraction would be higher, i.e. the same absolute amount is stored, so model results are not affected. In this respect, it is interesting to observe the relatively slow decline in fetal vein concentrations after fetal ${ }^{3} \mathrm{H}$ glibenclamide perfusion is stopped, in absence of BSA (Fig. 5), which could indicate a role for the stroma in storage and the notion that a single well-mixed tissue compartment equally accessible from both sides may be too simplistic to capture the dynamics.

\section{Permeability}

The overall glibenclamide diffusive permeability surface area product per cotyledon was estimated to be $P S_{e f f}=3.5 \pm 1.3 \times 10^{-3} \mathrm{~L} / \mathrm{min}$ for the alternating fetal-maternal perfusion experiment (Fig. 5), which could be considered most representative for bidirectional transfer under a range of conditions. Similar values for $P S_{\text {eff }}$ were found for the separate fetal to maternal (Fig. 2) and maternal to fetal experiments (Fig. 7), further confirming the diffusive nature of transfer. Relative values for the MVM and BM permeability surface area product could be estimated with less confidence (larger variation), as distinguishing between their contributions to the overall permeability relies on non-steady state behaviour, e.g. due to tissue accumulation. Nonetheless, the MVM permeability surface area product was higher than for the BM, consistent with our previous studies for cortisol [43], fatty acids [44] and amino acids [32]. This may reflect the larger surface area of the MVM and the fact that the estimated permeability for the BM would incorporate any resistance of the stroma and endothelium. Glibenclamide is lipophilic $\log D_{7.4}=2.1$ [42], supporting its transfer by membrane diffusion. It was previously concluded that the placental transfer of glibenclamide compared to other compounds is in line with its physiochemical properties once medium protein binding is taken into account $[12,16]$.

The model assumed well-mixed compartments as a first approximation, which is a clear simplification of the actual maternal and fetal circulations. Increasing or decreasing compartmental volume fractions had a small effect on dynamics, but did not affect steady states and effective permeability estimates. Experimental 
results for individual cotyledons displayed a large variation in maternal and fetal vein concentrations. Normalising uptake and transfer by cotyledon weight did not generally reduce variation, indicating that overall weight is not necessarily directly representative of the regions that are effectively perfused in the experiments. Nor was there a clear correlation with the permeability surface area product for creatinine transfer.

The effective permeability surface area product $\left(P S_{e f f}=3.5 \times 10^{-3} \mathrm{~L} / \mathrm{min}\right)$ from the experiments can be multiplied by the number of cotyledons to estimate an order of magnitude for the total placental permeability and transfer. For example, based on 13 cotyledons [45], a therapeutic glibenclamide concentration of 0.4 $\mu \mathrm{mol} / \mathrm{L}$, high affinity dissociation constant $K_{d}=0.71 \mu \mathrm{mol} / \mathrm{L}$ [39] and human albumin concentration of $29 \mathrm{~g} / \mathrm{L}$ in pregnant women [16], the free fraction of glibenclamide is $0.16 \%$ and the maximum rate of placental transfer would be 30 $\mathrm{pmol} / \mathrm{min}$, with the fetal concentration set to zero. However, fetal concentrations in vivo will be determined by the rate of placental transfer in relation to the rate of fetal metabolism. In this respect, glibenclamide metabolites are more potent transporter substrates than glibenclamide itself [42] and their placental transfer will need to be determined. Glibenclamide metabolism in the placenta itself may be relatively limited [46]. Such questions can be more fully addressed by incorporating the current placental model in larger modelling frameworks including the full maternal and fetal circulations in vivo [47-49].

\section{Summary and conclusions}

Based on the experimental and modelling results presented, we conclude that albumin binding and not transporter activity, is the dominant factor in the transfer of glibenclamide across the human placenta. The effect of BSP binding to albumin on promoting the diffusive transfer of glibenclamide highlights the importance of drugprotein binding interactions and their evaluation using computational modelling. In addition, accumulation over time due to high levels of tissue binding needs to be considered in the interpretation of perfusion experiments and their translation to the physiological situation in vivo. 
Author contributions: EL and GH performed experimental work and BS performed computational modelling. RL, BS and JC guided the design and performances of specific aspects of the experiments. BS, RL and JC conceived the study. EL, BG, JC and RL wrote the manuscript and all authors contributed to its revision and final form.

Declaration of interests: None.

Acknowledgements: This work was funded by the BBSRC (BB/R002762/1). We would like to thank the midwives at the princess Anne Hospital for their assistance in collecting placentas. 


\section{References}

1. Cleal, J.K., et al., A systems perspective on placental amino acid transport. J Physiol, 2018. 596(23): p. 5511-5522.

2. Staud, F., L. Cerveny, and M. Ceckova, Pharmacotherapy in pregnancy; effect of ABC and SLC transporters on drug transport across the placenta and fetal drug exposure. J Drug Target, 2012. 20(9): p. 736-63.

3. Gedeon, C., et al., Transport of glyburide by placental ABC transporters: implications in fetal drug exposure. Placenta, 2006. 27(11-12): p. 1096-102.

4. Han, L.W., C. Gao, and Q. Mao, An update on expression and function of $P$ gp/ABCB1 and BCRP/ABCG2 in the placenta and fetus. Expert Opin Drug Metab Toxicol, 2018. 14(8): p. 817-829.

5. Vahakangas, K. and P. Myllynen, Drug transporters in the human bloodplacental barrier. Br J Pharmacol, 2009. 158(3): p. 665-78.

6. St-Pierre, M.V., et al., Expression of members of the multidrug resistance protein family in human term placenta. Am J Physiol Regul Integr Comp Physiol, 2000. 279(4): p. R1495-503.

7. Gedeon, C., et al., Investigating the potential role of multi-drug resistance protein (MRP) transporters in fetal to maternal glyburide efflux in the human placenta. J Obstet Gynaecol, 2008. 28(5): p. 485-9.

8. Atkinson, D.E., et al., Role of MDR1 and MRP1 in trophoblast cells, elucidated using retroviral gene transfer. Am J Physiol Cell Physiol, 2003. 285(3): p. C584-91.

9. Grube, M., et al., Organic anion transporting polypeptide 2B1 and breast cancer resistance protein interact in the transepithelial transport of steroid sulfates in human placenta. Drug Metab Dispos, 2007. 35(1): p. 30-5.

10. Lofthouse, E.M., et al., Glutamate cycling may drive organic anion transport on the basal membrane of human placental syncytiotrophoblast. J Physiol, 2015. 593(20): p. 4549-59.

11. Schwartz, R.A., et al., Glyburide transport across the human placenta. Obstet Gynecol, 2015. 125(3): p. 583-8.

12. Koren, G., Glyburide and fetal safety; transplacental pharmacokinetic considerations. Reprod Toxicol, 2001. 15(3): p. 227-9.

13. Farrar, D., et al., Treatments for gestational diabetes: a systematic review and meta-analysis. BMJ open, 2017. 7(6): p. e015557.

14. Langer, O., et al., A comparison of glyburide and insulin in women with gestational diabetes mellitus. N Engl J Med, 2000. 343(16): p. 1134-8.

15. Elliott, B.D., et al., Insignificant transfer of glyburide occurs across the human placenta. Am J Obstet Gynecol, 1991. 165(4 Pt 1): p. 807-12.

16. Nanovskaya, T.N., et al., Effect of human serum albumin on transplacental transfer of glyburide. Biochem Pharmacol, 2006. 72(5): p. 632-9.

17. Kraemer, J., et al., Perfusion studies of glyburide transfer across the human placenta: implications for fetal safety. Am J Obstet Gynecol, 2006. 195(1): p. 270-4.

18. Pollex, E., A. Lubetsky, and G. Koren, The role of placental breast cancer resistance protein in the efflux of glyburide across the human placenta. Placenta, 2008. 29(8): p. 743-7.

19. Nanovskaya, T.N., et al., Effect of albumin on transplacental transfer and distribution of rosiglitazone and glyburide. The journal of maternal-fetal \& neonatal medicine : the official journal of the European Association of 
Perinatal Medicine, the Federation of Asia and Oceania Perinatal Societies, the International Society of Perinatal Obstetricians, 2008. 21(3): p. 197207.

20. Gedeon, C., et al., Breast cancer resistance protein: mediating the transplacental transfer of glyburide across the human placenta. Placenta, 2008. 29(1): p. 39-43.

21. Hemauer, S.J., et al., Role of human placental apical membrane transporters in the efflux of glyburide, rosiglitazone, and metformin. American journal of obstetrics and gynecology, 2010. 202(4): p. 383.e1-7.

22. Cygalova, L.H., et al., Transplacental pharmacokinetics of glyburide, rhodamine 123, and BODIPY FL prazosin: effect of drug efflux transporters and lipid solubility. J Pharmacol Exp Ther, 2009. 331(3): p. 1118-25.

23. Pollex, E.K., et al., Breast cancer resistance protein (BCRP)-mediated glyburide transport: effect of the C421A/Q141K BCRP single-nucleotide polymorphism. Drug metabolism and disposition: the biological fate of chemicals, 2010. 38(5): p. 740-4.

24. Yu, J., et al., Intestinal Drug Interactions Mediated by OATPs: A Systematic Review of Preclinical and Clinical Findings. Journal of pharmaceutical sciences, 2017. 106(9): p. 2312-2325.

25. Mitra, P. and K.L. Audus, MRP isoforms and BCRP mediate sulfate conjugate efflux out of BeWo cells. Int J Pharm, 2010. 384(1-2): p. 15-23.

26. Greupink, R., et al., Semi-mechanistic physiologically-based pharmacokinetic modeling of clinical glibenclamide pharmacokinetics and drug-druginteractions. Eur J Pharm Sci, 2013. 49(5): p. 819-28.

27. Koenen, A., et al., Steroid hormones specifically modify the activity of organic anion transporting polypeptides. Eur J Pharm Sci, 2012. 47(4): p. 774-80.

28. Varma, M.V., et al., Mechanism-based pharmacokinetic modeling to evaluate transporter-enzyme interplay in drug interactions and pharmacogenetics of glyburide. AAPS J, 2014. 16(4): p. 736-48.

29. Schneider, H., M. Panigel, and J. Dancis, Transfer across the perfused human placenta of antipyrine, sodium and leucine. Am J Obstet Gynecol, 1972. 114(6): p. 822-8.

30. Cleal, J.K., et al., Facilitated transporters mediate net efflux of amino acids to the fetus across the basal membrane of the placental syncytiotrophoblast. J Physiol, 2011. 589(Pt 4): p. 987-97.

31. Panitchob, N., et al., Computational modelling of placental amino acid transfer as an integrated system. Biochim Biophys Acta, 2016. 1858(7 Pt A): p. 1451-61.

32. Sengers, B.G., C.P. Please, and R.M. Lewis, Computational modelling of amino acid transfer interactions in the placenta. Exp Physiol, 2010. 95(7): p. 82940 .

33. Mayhew, T.M., A stereological perspective on placental morphology in normal and complicated pregnancies. J Anat, 2009. 215(1): p. 77-90.

34. Proks, P., et al., Binding of sulphonylureas to plasma proteins - A KATP channel perspective. PLoS One, 2018. 13(5): p. e0197634.

35. Cheng, F.Q., et al., Fluorescence study on the interaction of human serum albumin with bromsulphalein. Spectrochim Acta A Mol Biomol Spectrosc, 2006. 65(5): p. 1144-7. 
36. Karttunen, V., et al., Criteria and challenges of the human placental perfusion - Data from a large series of perfusions. Toxicol In Vitro, 2015. 29(7): p. 1482-91.

37. Matsson, P., et al., Identification of novel specific and general inhibitors of the three major human ATP-binding cassette transporters P-gp, BCRP and MRP2 among registered drugs. Pharm Res, 2009. 26(8): p. 1816-31.

38. Karlgren, M., et al., Classification of inhibitors of hepatic organic anion transporting polypeptides (OATPs): influence of protein expression on drugdrug interactions. J Med Chem, 2012. 55(10): p. 4740-63.

39. Matsuda, R., et al., Analysis of drug interactions with modified proteins by high-performance affinity chromatography: binding of glibenclamide to normal and glycated human serum albumin. J Chromatogr A, 2012. 1265: p. 114-22.

40. Crooks, M.J. and K.F. Brown, The binding of sulphonylureas to serum albumin. J Pharm Pharmacol, 1974. 26(5): p. 304-11.

41. Hebert, M.F., et al., Are we optimizing gestational diabetes treatment with glyburide? The pharmacologic basis for better clinical practice. Clin Pharmacol Ther, 2009. 85(6): p. 607-14.

42. Li, R., et al., Transporter-Mediated Disposition, Clinical Pharmacokinetics and Cholestatic Potential of Glyburide and Its Primary Active Metabolites. Drug Metab Dispos, 2017. 45(7): p. 737-747.

43. Stirrat, L.I., et al., Transfer and Metabolism of Cortisol by the Isolated Perfused Human Placenta. J Clin Endocrinol Metab, 2018. 103(2): p. 640648.

44. Perazzolo, S., et al., The influence of placental metabolism on fatty acid transfer to the fetus. J Lipid Res, 2017. 58(2): p. 443-454.

45. Barker, D., et al., Maternal cotyledons at birth predict blood pressure in childhood. Placenta, 2013. 34(8): p. 672-5.

46. Ravindran, S., et al., Identification of glyburide metabolites formed by hepatic and placental microsomes of humans and baboons. Biochemical pharmacology, 2006. 72(12): p. 1730-7.

47. De Sousa Mendes, M., et al., Prediction of human fetal pharmacokinetics using ex vivo human placenta perfusion studies and physiologically based models. Br J Clin Pharmacol, 2016. 81(4): p. 646-57.

48. Ke, A.B., R. Greupink, and K. Abduljalil, Drug Dosing in Pregnant Women: Challenges and Opportunities in Using Physiologically Based Pharmacokinetic Modeling and Simulations. CPT Pharmacometrics Syst Pharmacol, 2018. 7(2): p. 103-110.

49. Schalkwijk, S., et al., Prediction of Fetal Darunavir Exposure by Integrating Human Ex-Vivo Placental Transfer and Physiologically Based Pharmacokinetic Modeling. Clin Pharmacokinet, 2018. 57(6): p. 705-716. 


\section{FIGURES}

A)

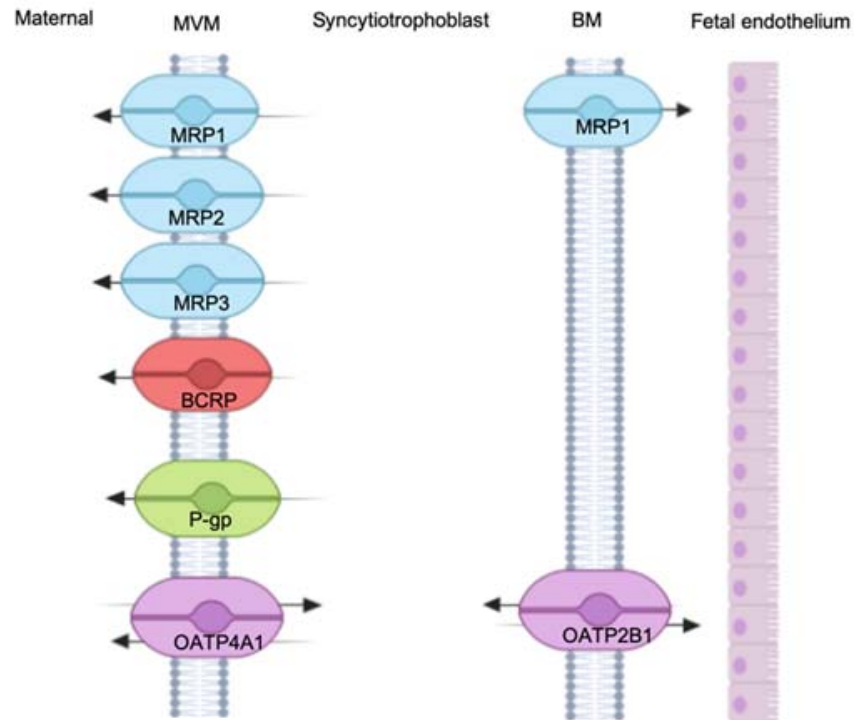

B)

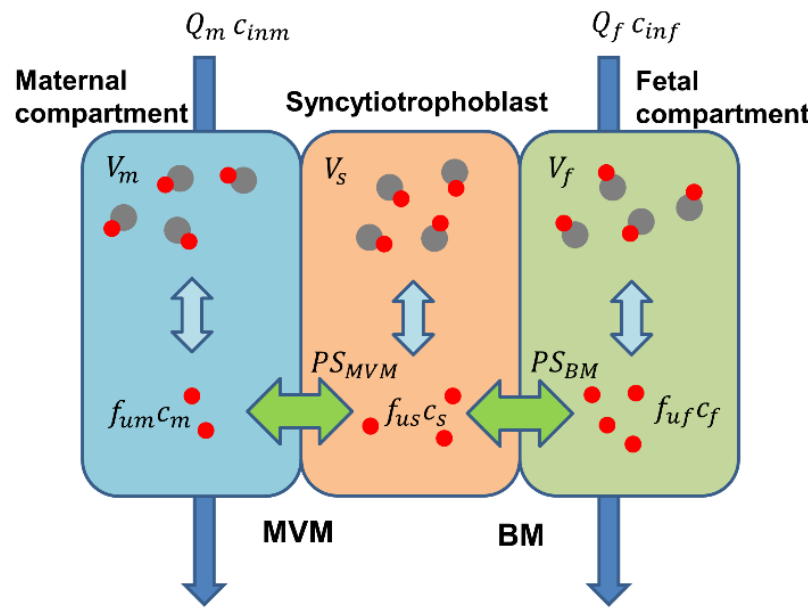

Figure 1: Overview of placental transfer. A) Reported localisation of relevant transporters on the apical maternal facing microvillous membrane (MVM) and fetal facing basal membrane (BM). B) Compartmental model for placental transfer, consisting of three separate maternal, syncytiotrophoblast and fetal volumes. Within each compartment an equilibrium exists between glibenclamide bound to BSA and a free, unbound, fraction (light blue arrows). Exchange between compartments across the MVM and BM was assumed to be governed by simple diffusion determined by the free concentrations (green arrows). 
A)

B)

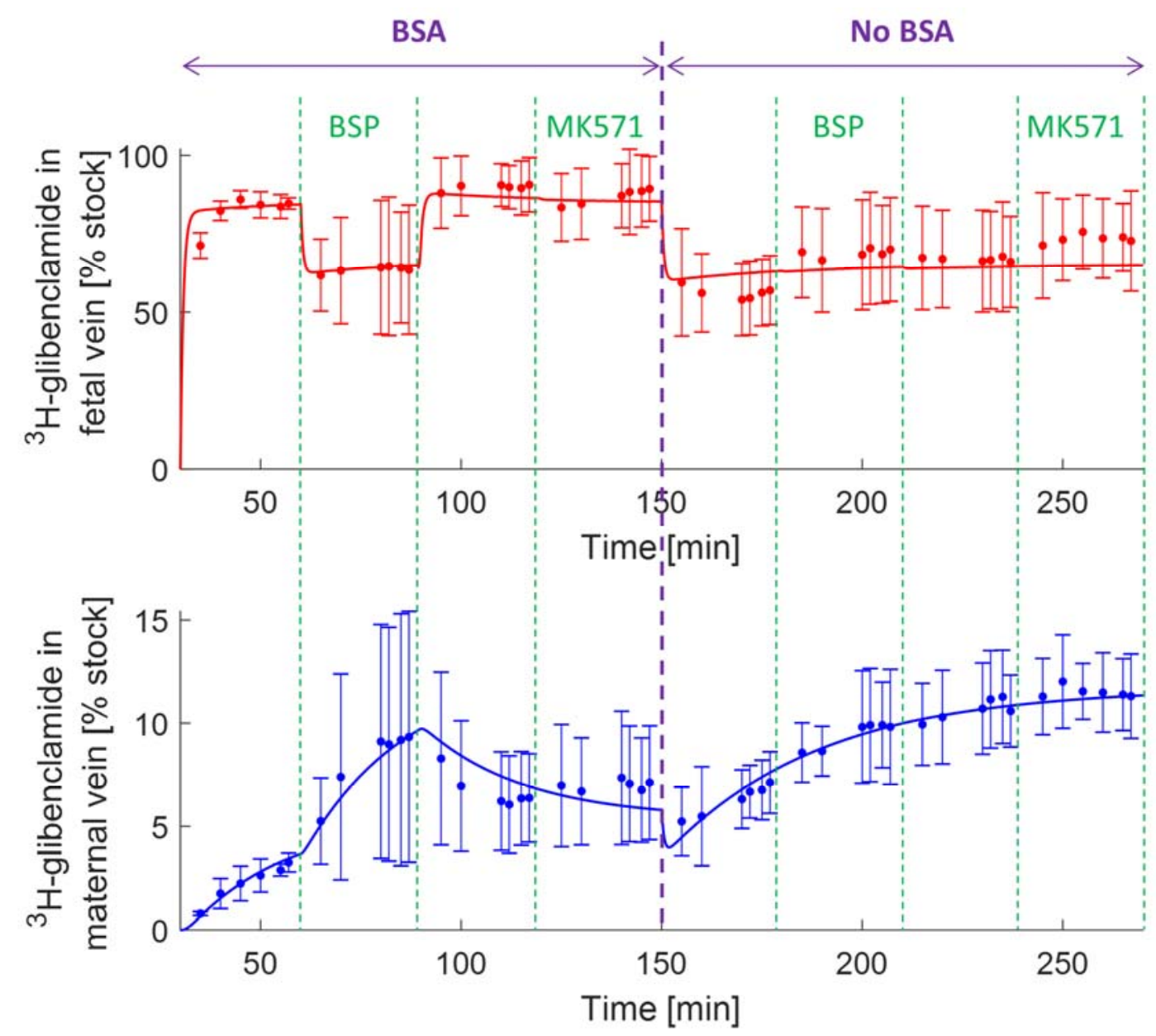

Figure 2: Fetal side glibenclamide perfusion. Summary data for total ${ }^{3} \mathrm{H}$-glibenclamide levels (bound + unbound) in the fetal vein (A) and transfer into the maternal vein (B). Data presented as mean \pm SD of three placentas. Solid lines represent computational model results. Results for individual placentas are included in Supplementary data D. 

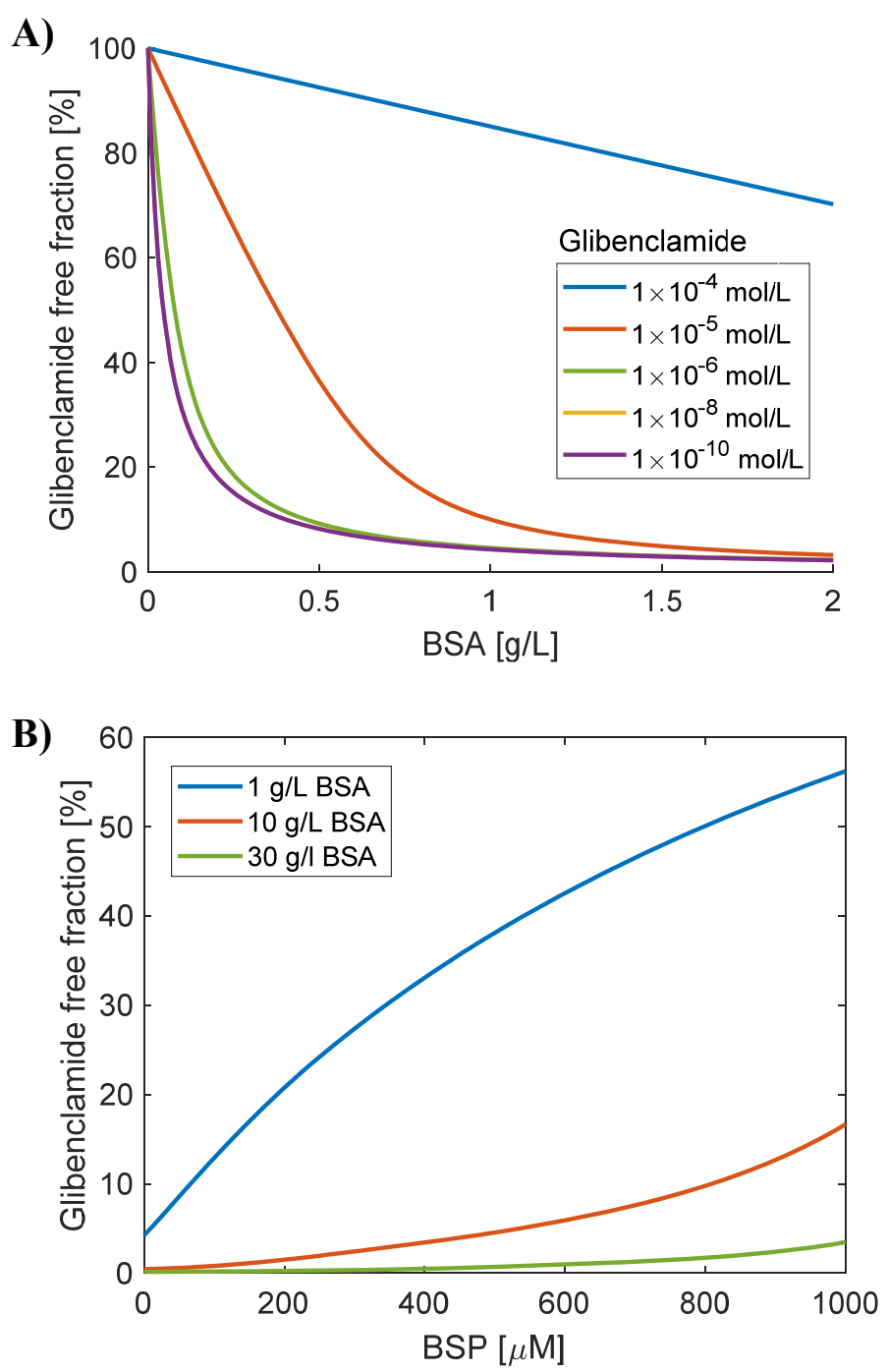

Figure 3: Theoretical predictions of glibenclamide binding. A) Glibenclamide free fraction as a function of BSA. For low levels of glibenclamide the free fraction becomes independent of glibenclamide concentration, as can be seen from the overlap between curves. Note high glibenclamide concentrations would exceed solubility in absence of BSA. B) BSP was predicted to increase the free fraction of glibenclamide by binding to BSA. Results for tracer levels of glibenclamide (93 pmol/L). $1 \mathrm{~g} / \mathrm{L} \mathrm{BSA}$ was used in the experiments. 


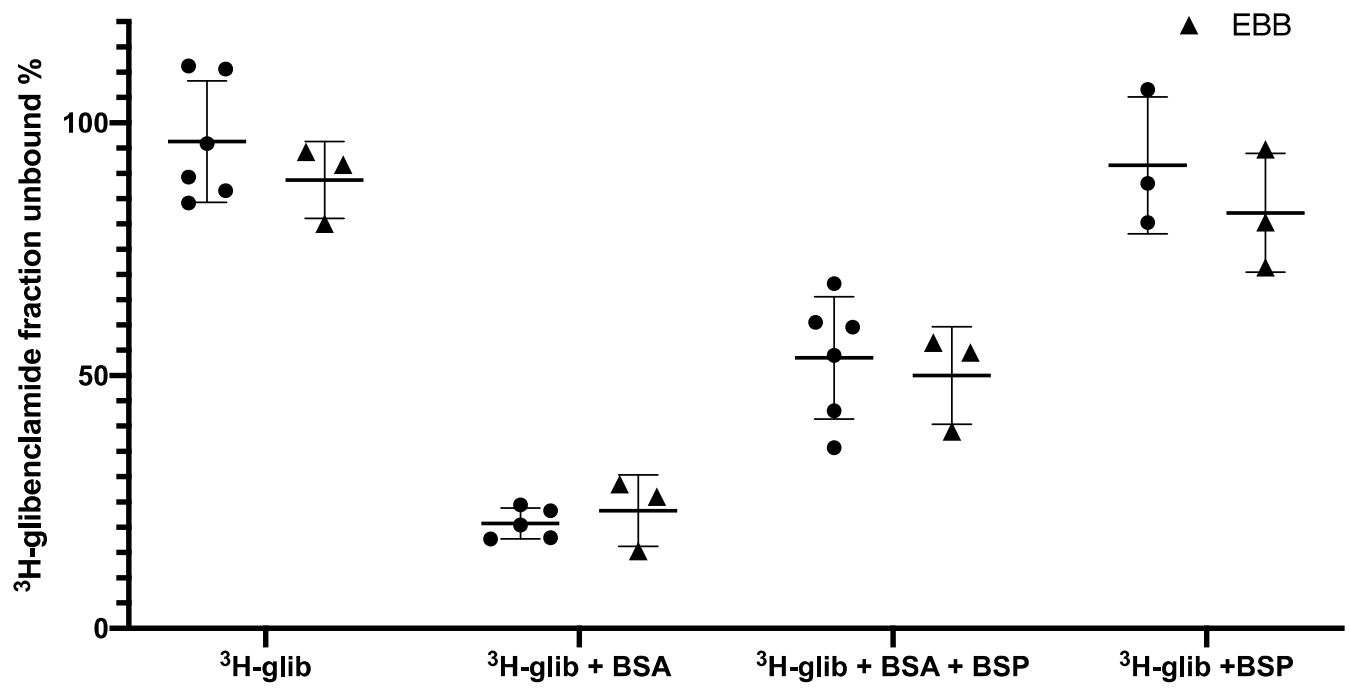

Figure 4: Free fractions of ${ }^{3} \mathrm{H}$-glibenclamide in EBB buffer and fetal perfusate from the fetal side glibenclamide perfusion experiments (Fig. 2). Results presented as individual data points and mean $\pm \operatorname{SD}(n=3-6)$. 
A)

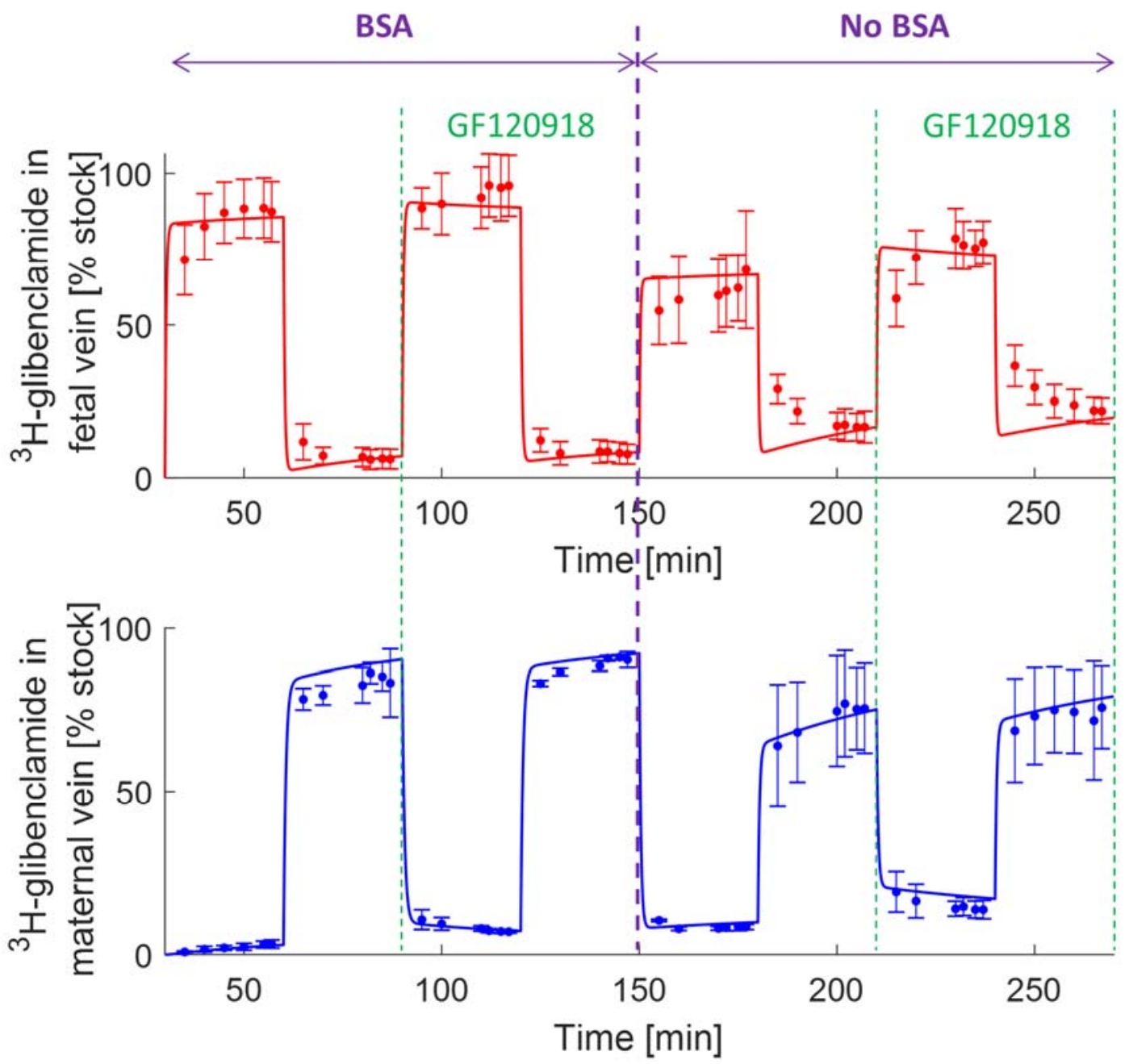

Figure 5: Alternating fetal / maternal glibenclamide perfusion. Summary data showing total ${ }^{3} \mathrm{H}$-glibenclamide levels (bound + unbound) in the fetal vein (A) and maternal vein (B). Fetal and maternal "high blocks" correspond to tracer perfusion in the respective circulations. Experimental data presented as mean \pm SD of three placentas. Solid lines represent model results. Results for individual placentas are included in Supplementary data D. 

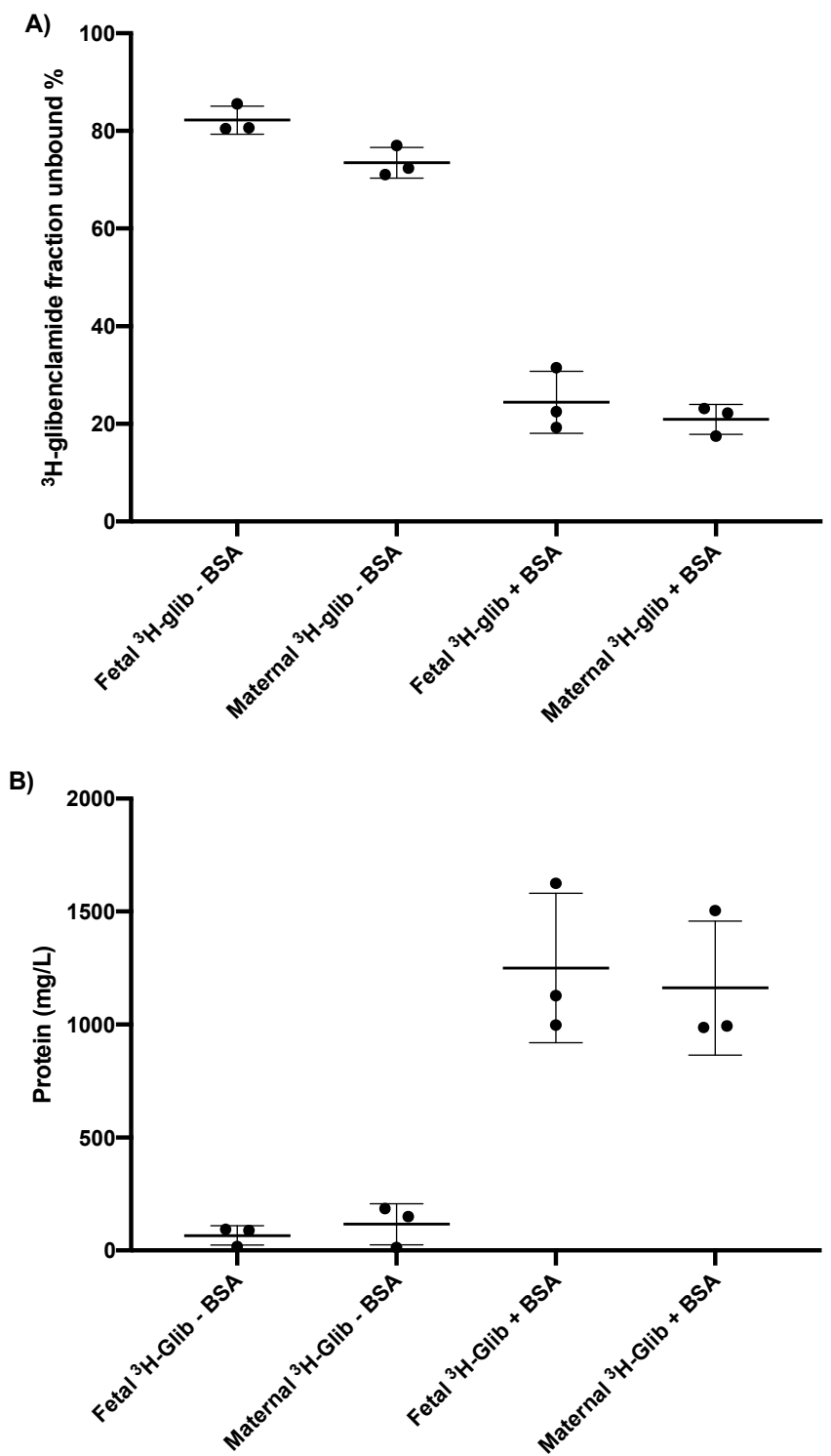

Figure 6: Maternal and fetal glibenclamide free fractions and protein concentrations. A) Free fractions in perfusate from the alternating fetal / maternal glibenclamide perfusion experiments. B) Measured protein concentrations in the corresponding samples. All data presented as individual data points and mean \pm SD of three placentas. 
A)

No BSA

)

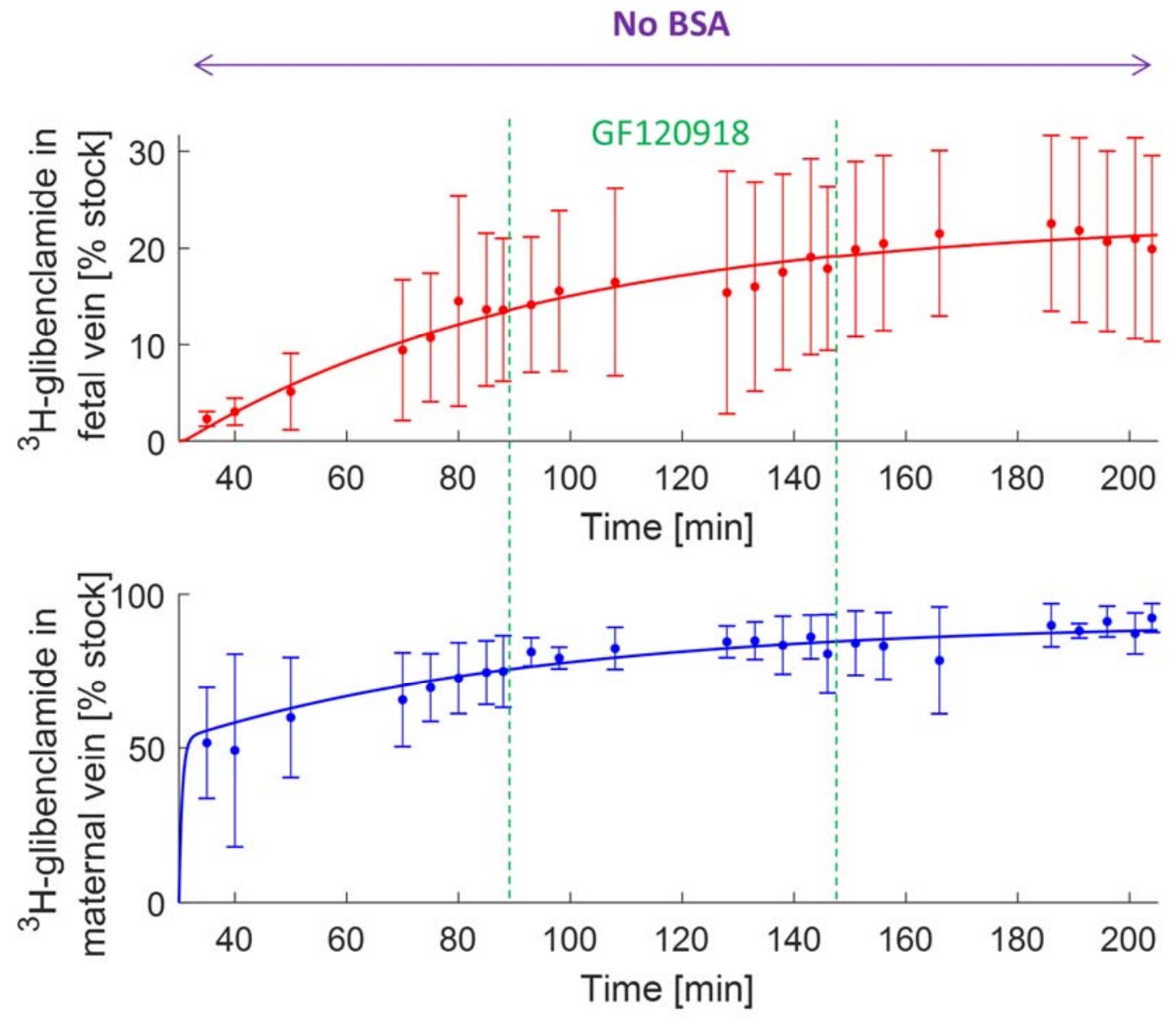

Figure 7: Maternal side glibenclamide perfusion. Summary data showing total ${ }^{3} \mathrm{H}-$ glibenclamide levels (bound + unbound) in the fetal vein (A) and maternal vein (B). Experimental data presented as mean $\pm \mathrm{SD}$ of three placentas. Solid lines represent model results. Results for individual placentas are included in Supplementary data D. 


\section{$\underline{\text { Supplementary data }}$}

\section{Glibenclamide transfer across the perfused human placenta is determined by}

\section{albumin binding not transporter activity}

Emma M. Lofthouse, Jane Cleal, Georgina Hudson, Rohan M. Lewis,

Bram G. Sengers

\section{Supplementary data A: Creatinine model}

The placental transfer of creatinine was assumed to be governed by simple paracellular diffusion between the maternal and fetal compartments (Fig. S1).

$\begin{array}{ll}\frac{\mathrm{d} c_{m \text { crea }}}{\mathrm{d} t}=\frac{1}{V_{m}}\left(P S_{\text {crea }}\left(c_{f \text { crea }}-c_{\text {m crea }}\right)+Q_{m}\left(c_{\text {inm crea }}-c_{m \text { crea }}\right)\right) & \text { Eq. S1 } \\ \frac{\mathrm{d} c_{f \text { crea }}}{\mathrm{d} t}=\frac{1}{V_{f}}\left(P S_{\text {crea }}\left(c_{m \text { crea }}-c_{f \text { crea }}\right)+Q_{f}\left(c_{\text {inf crea }}-c_{f \text { crea }}\right)\right) & \text { Eq. S2 }\end{array}$

Here $c_{m \text { crea }}$ and $c_{f \text { crea }}[\mathrm{mol} / \mathrm{L}]$ are the creatinine concentrations in the maternal and fetal compartments, respectively, while $V[\mathrm{~L}]$ are the compartment volumes. $c_{\text {inm }}$ crea and $c_{\text {inf crea }}$ are the creatinine arterial inlet concentrations in the maternal and fetal compartments. $P S_{\text {crea }}[\mathrm{L} / \mathrm{min}]$ is the overall cotyledon permeability surface area product for creatinine. $Q[\mathrm{~L} / \mathrm{min}]$ are the perfusion flow rates for the maternal and fetal circulation.

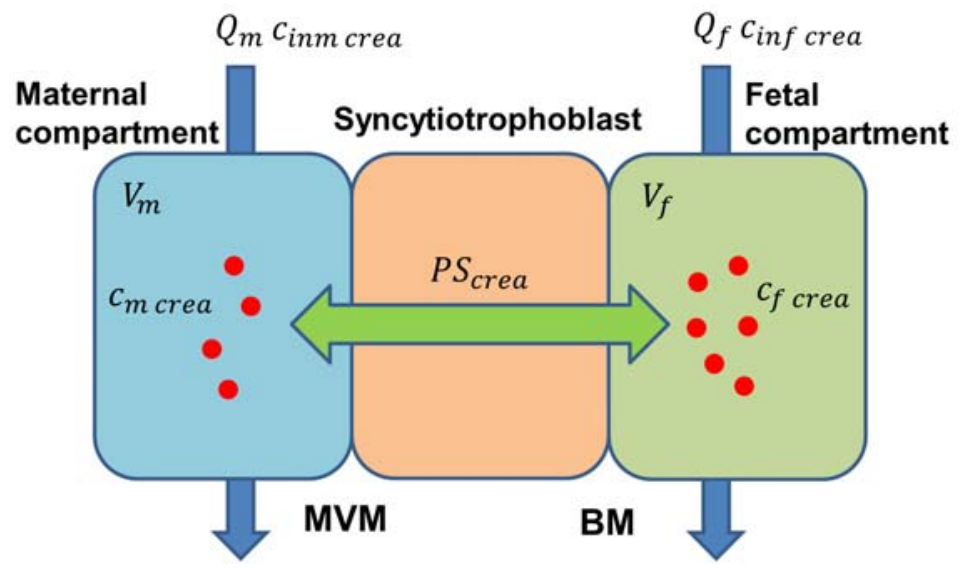

Figure S1: Creatinine model schematic. Creatinine transfer was described by simple paracellular diffusion between the maternal and fetal compartments, bypassing the syncytiotrophoblast. 


\section{Supplementary data B: Free fraction calculation}

The glibenclamide free fraction $f_{u} g l b[-]$ in presence of BSA was calculated by solving the standard quadratic equation for the unbound glibenclamide concentration $c_{g l b}{ }^{F}[\mathrm{~mol} / \mathrm{L}]$ derived from the binding equilibrium relationship, assuming a single binding site:

$$
c_{g l b}{ }^{F}=\frac{1}{2}\left(-K_{d g l b}+c_{g l b}{ }^{T}-c_{B S A}{ }^{T} \pm \sqrt{\left(K_{d g l b}-c_{g l b}{ }^{T}+c_{B S A}{ }^{T}\right)^{2}+4\left(K_{d g l b} c_{g l b}{ }^{T}\right)}\right) \quad \text { Eq. S3 }
$$

Here $c_{g l b}{ }^{T}$ and $c_{B S A}{ }^{T}[\mathrm{~mol} / \mathrm{L}]$ are the total glibenclamide and BSA concentrations (bound + unbound), and $K_{d} g l b[\mathrm{~mol} / \mathrm{L}]$ the dissociation constant. The glibenclamide free fraction is then given by $f_{u g l b}=\frac{c_{g l b}{ }^{F}}{c_{g l b}{ }^{T}}$.

\section{Supplementary data C: Binding equilibrium for multiple substrates}

The binding equilibrium between BSA, glibenclamide and BSP was solved numerically using Newton iteration, starting from an initial estimate $c_{B S A}{ }^{F}$ for the free, unbound concentration of BSA. In the following, $c_{g l b}{ }^{T}, C_{B S P}{ }^{T}$ and $c_{B S A}{ }^{T}$ $[\mathrm{mol} / \mathrm{L}]$ represent the total concentrations (free + bound) of glibenclamide, BSP and BSA. The bound concentrations $c_{g l b}{ }^{B}$ and $c_{B S P}{ }^{B}$ are then given by:

$$
\begin{aligned}
c_{g l b}{ }^{B} & =\frac{c_{g l b}{ }^{T} c_{B S A}{ }^{F}}{K_{d g l b}+c_{B S A} F} \\
c_{B S P}{ }^{B} & =\frac{c_{B S P}{ }^{T} c_{B S A}{ }^{F}}{K_{d B S P}+c_{B S A}{ }^{F}}
\end{aligned}
$$

Since $c_{g l b}{ }^{B}$ and $c_{B S P}{ }^{B}$ are equal to the concentrations of BSA bound to either glibenclamide or BSP, the residual error $R$ in total BSA is equal to:

$$
R=c_{B S A}{ }^{T}-c_{B S A}{ }^{F}-c_{g l b}{ }^{B}-c_{B S P}{ }^{B}
$$

The derivative with respect to $C_{B S A F}$ is:

$$
K=1+\frac{c_{g l b}{ }^{T} K_{d} g l b}{\left(K_{d g l b}+c_{B S A}{ }^{F}\right)^{2}}+\frac{c_{B S P}{ }^{T} K_{d B S P}}{\left(K_{d B S P}+c_{B S A}{ }^{F}\right)^{2}}
$$

and a new estimate for $c_{B S A}{ }^{F}$ is then given by:

$$
\left(c_{B S A}^{F}\right)_{n+1}=\left(c_{B S A}^{F}\right)_{n}+\left(\frac{R}{K}\right)
$$


The procedure Eqs. S4-S8 was repeated until convergence was obtained, after which the free fraction of glibenclamide in presence of both BSA and BSP was calculated using the bound concentration from Eq. S4:

$$
f_{u g l b}=1-\frac{c_{B S A}{ }^{F}}{K_{d g l b}+c_{B S A}{ }^{F}}
$$

\section{Supplementary data D: Results for individual placentas}
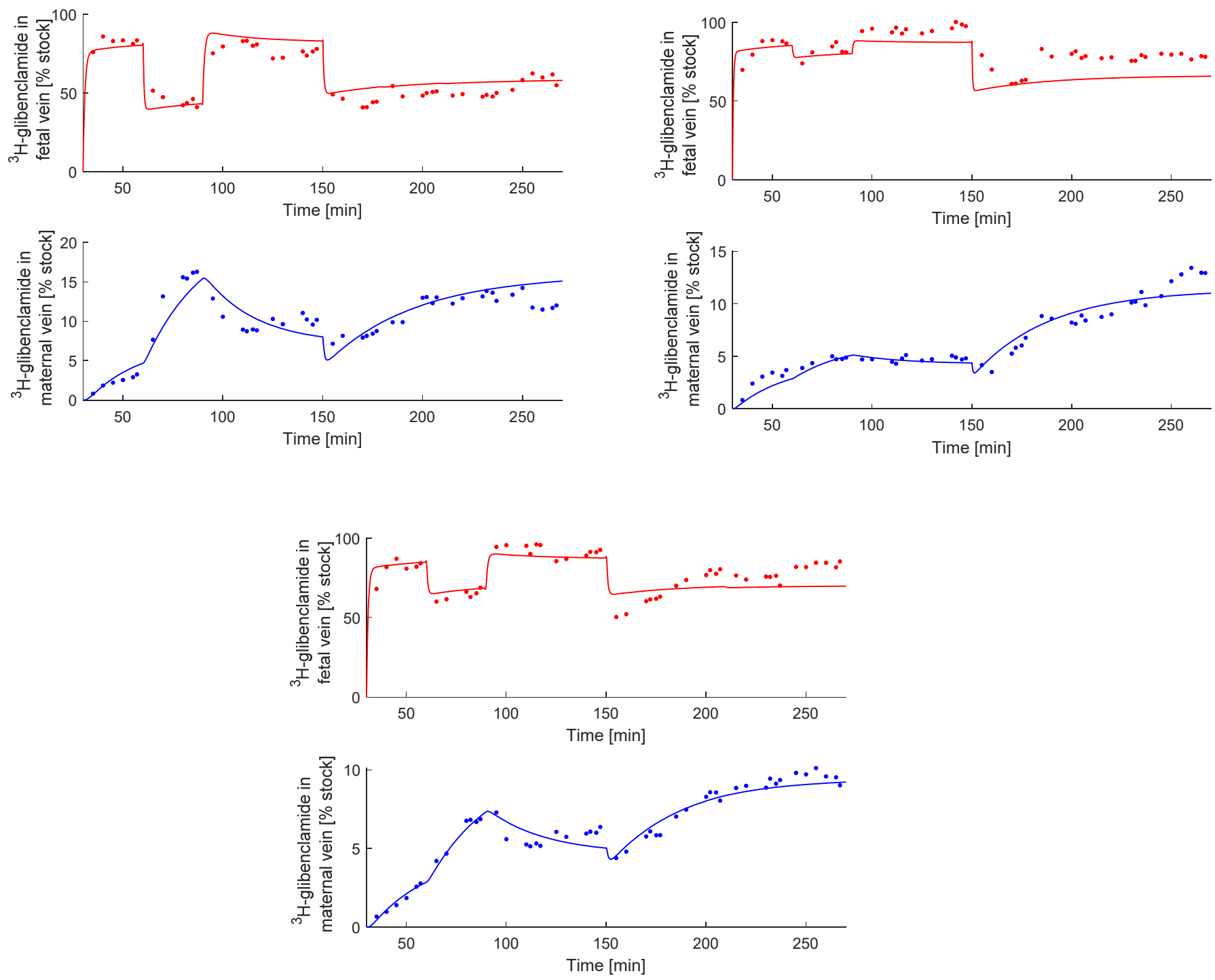

Figure S2: Fetal side ${ }^{3} \mathbf{H}$-glibenclamide perfusion. Individual data for each of the three placentas evaluated. Solid lines represent the results of the computational model for each placenta. 

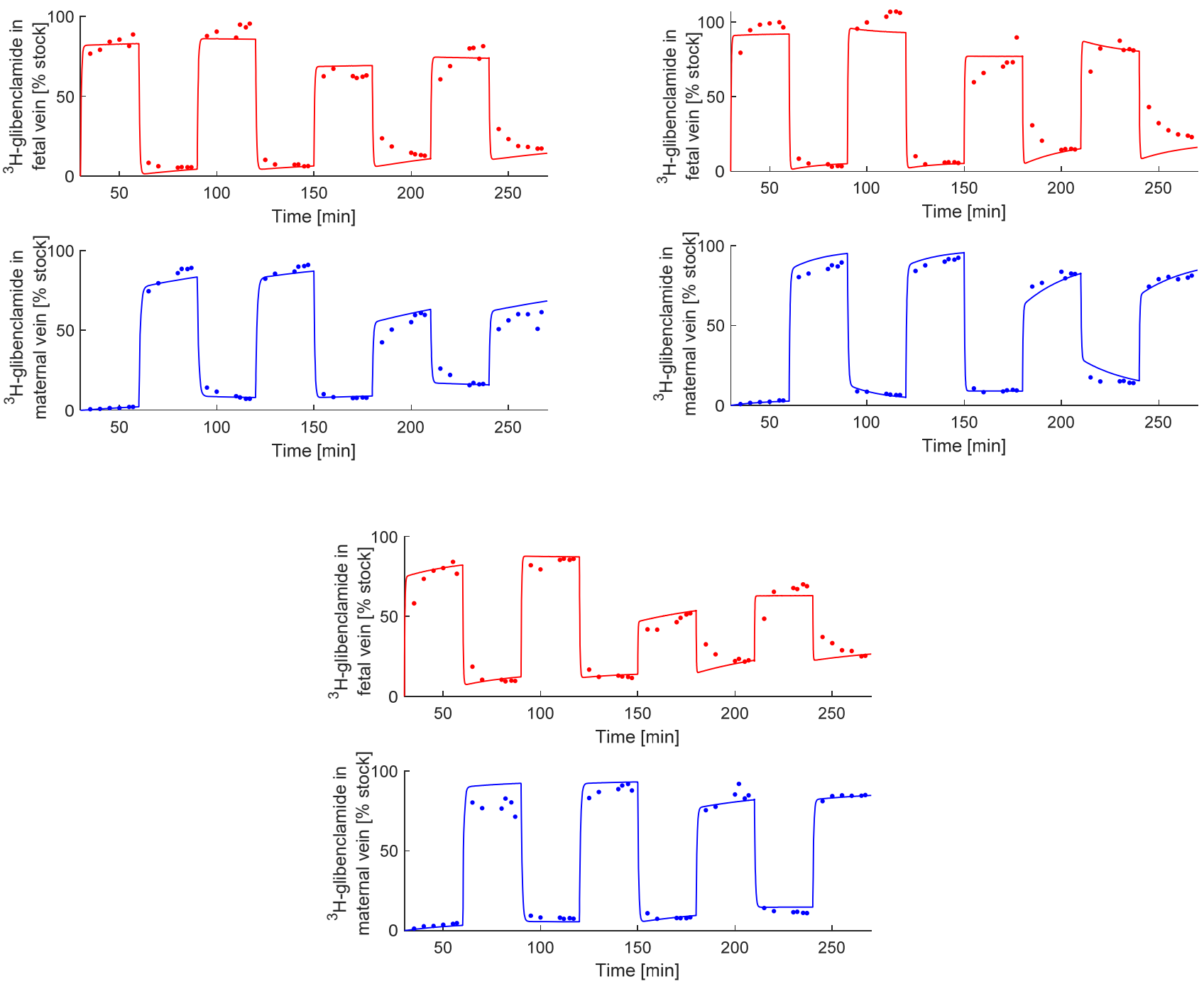

Figure S3: Alternating fetal / maternal glibenclamide perfusion. Individual data for each of the three placentas evaluated. Solid lines represent the results of the computational model for each placenta. 

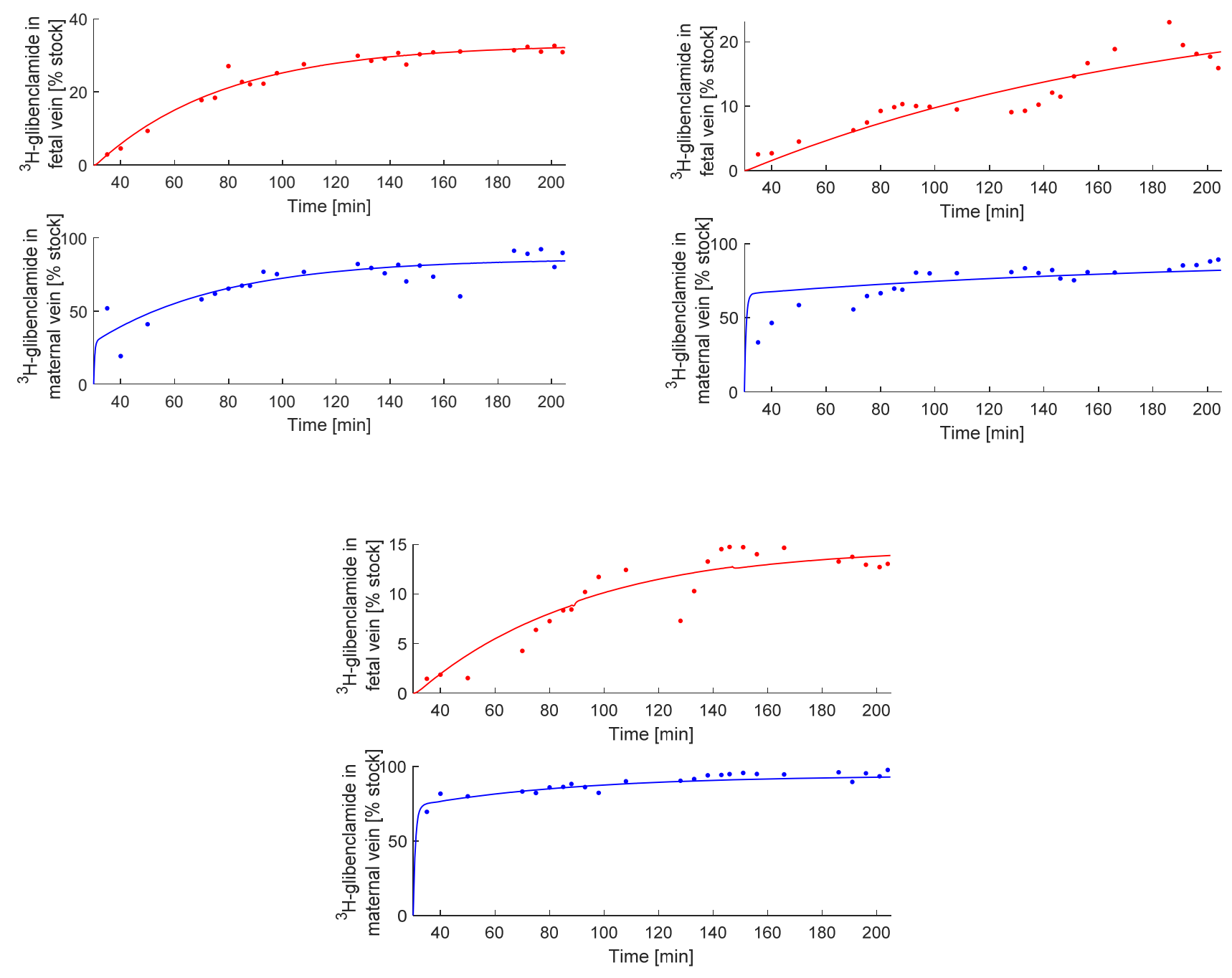

Figure S4: Maternal side glibenclamide perfusion. Individual data for each of the three placentas evaluated. Solid lines represent the results of the computational model for each placenta. 


\section{Supplementary data E: Closed loop simulations}

Closed loop perfusion simulations were performed based on the experiments by Navoskaya [16, 19], investigating the effect of albumin and native protein released on placental glibenclamide transfer. Two additional compartments were added to the model in Fig. 1B, representing the maternal and fetal reservoirs $(250$ and $150 \mathrm{~mL}$, respectively). Simulations were performed using the parameters from the alternating fetal-maternal perfusion experiments and a range of albumin concentrations was evaluated $(0,1,10$ and $30 \mathrm{~g} / \mathrm{L}$, equal in both circulations). Based on the published results [16], in all cases an additional contribution of $630 \mathrm{mg} / \mathrm{L}$ of native protein was included for the maternal circulation only. Free fractions were calculated using Eq. S3, assuming that the native protein binding properties were the same as for albumin. The glibenclamide dissociation constant used was $K_{d ~ g l b}=3.9 \mu \mathrm{mol} / \mathrm{L}$, based on our dialysis results for $\mathrm{EBB}+\mathrm{BSA}$, and calculated using Eq. S10, with $c_{g l b}{ }^{F}$ the free concentration:

$$
K_{d g l b}=\frac{\left(c_{g l b}\right)^{2}+c_{g l b}{ }^{F}\left(c_{B S A}{ }^{T}-c_{g l b}{ }^{T}\right)}{c_{g l b}{ }^{T}-c_{g l b}{ }^{F}}
$$

Results for fetal to maternal transfer (Fig. S5) produced qualitatively similar results as those from the published experiments $[16,19]$. In particular, for zero albumin an apparent asymmetry in transfer was observed, with maternal concentrations higher than fetal at equilibrium (Fig. S5A). The reason for this is that, while free fractions are in equilibrium, a higher total concentration of glibenclamide is contained in the maternal circulation due to the higher protein binding. This asymmetry becomes less apparent as the albumin concentration is increased and starts to dominate the effect of any native protein in the experiment, so that the maternal and fetal concentrations approach a common equilibrium. When high, physiologically representative, albumin levels are added equally to both circulations, the transfer within the time scale of the experiment becomes limited, but continues at a very slow rate (Fig. S5C). 
A)

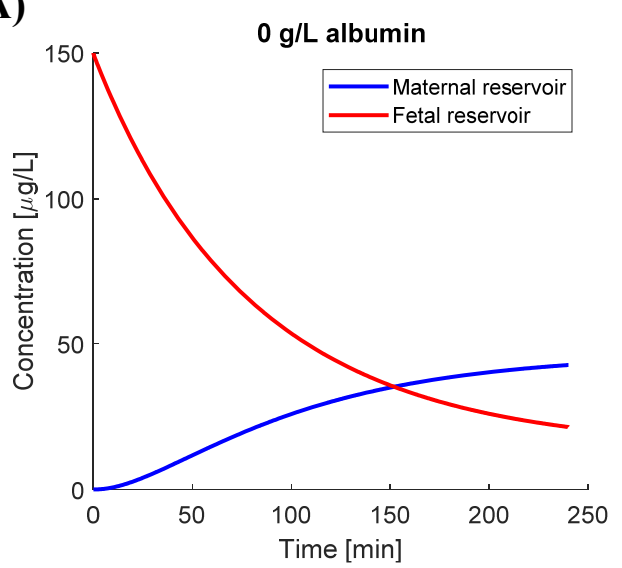

B)

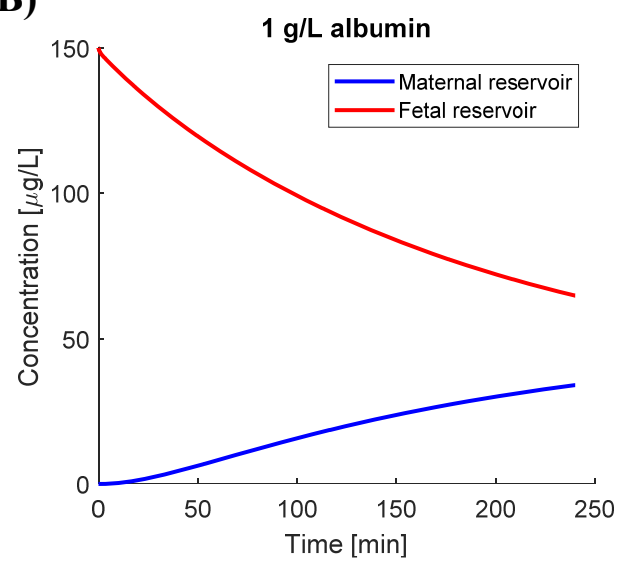

C)

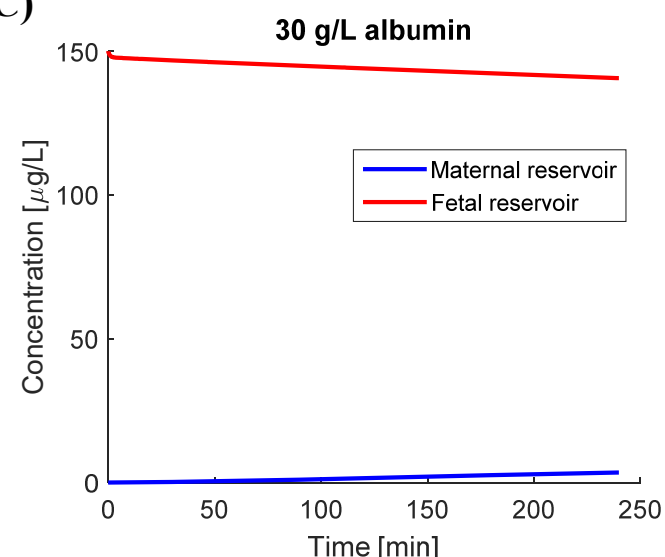

Figure S5: Model simulations of closed loop placental perfusion experiments based on Nanovskaya et al. $[16,19]$. A) Glibenclamide was added initially to the fetal circulation. At equilibrium, maternal concentrations were higher than fetal, due to the presence of additional native binding protein in the maternal circulation. B) This apparent asymmetry is lost as more and more albumin is added equally to both circulations, while transfer continued at a very slow rate for physiological albumin concentrations $\mathrm{C}$ ). 